\title{
Thermal deflections in multilayer microstructures and athermalization
}

Hamdi Torun and Hakan Urey

Citation: Journal of Applied Physics 100, 023527 (2006); doi: 10.1063/1.2216789

View online: http://dx.doi.org/10.1063/1.2216789

View Table of Contents: http://aip.scitation.org/toc/jap/100/2

Published by the American Institute of Physics

\section{A $|\mathrm{P}|$ Journal of Applied Physics}

Save your money for your research. It's now FREE to publish with us no page, color or publication charges apply.

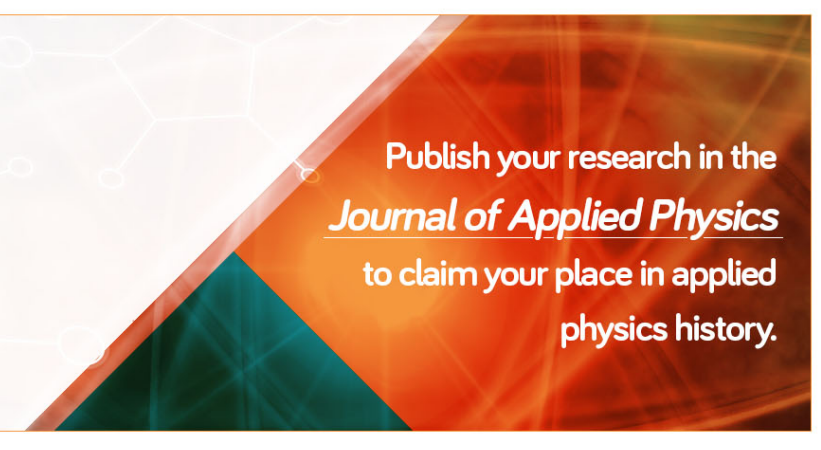




\title{
Thermal deflections in multilayer microstructures and athermalization
}

\author{
Hamdi Torun and Hakan Urey ${ }^{\text {a) }}$ \\ Koç University, Sariyer, Istanbul 34450, Turkey
}

(Received 13 November 2005; accepted 17 May 2006; published online 26 July 2006)

\begin{abstract}
Exact and approximate analytical solutions are developed for calculating the thermally induced deformation of three-layer cantilever structures. The solution is derived from the closed-form solutions for multilayer films. Thermal deformation and athermalization conditions are derived using dimensionless parameters for film to substrate thickness ratios for three-layer structures. The analytical solution for a narrow beam is applied to a scan mirror plate suspended with two torsional flexures. The results agreed well with finite element method simulations and experiments. Tests are performed using a bulk-micromachined silicon microelectromechanical system scanner that has a thin gold $(\mathrm{Au})$ coil layer on one side and an aluminum $(\mathrm{Al})$ mirror layer on the other side. Useful figures using film-to-substrate thickness ratios and the material independent normalized parameters are introduced for easy thermal deformation computations and performance trades for three-layer structures. () 2006 American Institute of Physics. [DOI: 10.1063/1.2216789]
\end{abstract}

\section{INTRODUCTION}

Many micromachined structures are formed by adding layers of materials on the top and bottom of a substrate wafer. Coefficients of thermal expansion (CTEs) mismatch between those layers can produce large stress and strains and deform the structure. Thermal deformation can have a significant effect in the device performance, particularly in optical microelectromechanical system (MEMS) applications where optical grade surface flatness is required ${ }^{1}$ and in applications that employ an array of microstructures where the array uniformity is critical. ${ }^{2,3}$ CTE mismatch problems are often encountered in silicon MEMS devices that employ metallic films due to low CTE of silicon. On the other hand, thermal deformations can also be used for sensing infrared radiation by converting the induced temperature difference into a mechanical deformation on a microcantilever structure. ${ }^{4}$

Thermal deformation for two layers can be found in the literature. ${ }^{5}$ An exact closed-form solution for stress distribution in multilayer systems subjected to both residual stresses and external bending was formulated by Hsueh. ${ }^{6}$ Closedform solutions for the deflection in bilayer and multilayer structures are reported in several references using different methods $^{7-9}$ but experimental verifications and comparison with finite element modeling results are often missing.

In this paper we derived exact formulas and simple approximations for the thermal deformation of three-layer structures and verified the results using finite element method (FEM) and experimental results. One important feature of the three-layer structure is the ability to make the structure athermal by proper material selection. A bulkmicromachined silicon $(\mathrm{Si})$ microscanner with an aluminum top layer and a gold bottom layer is used as a test case. Contour plots are obtained using normalized thickness ratios that allow making performance trades for the $\mathrm{Au}, \mathrm{Si}$, and $\mathrm{Al}$ material combination and also for arbitrary three materials.

${ }^{a)}$ Electronic mail: hurey@ku.edu.tr

\section{ANALYTICAL RESULTS FOR TRILAYER DEFLECTION}

Figure 1 shows a general multilayer structure anchored from one end. Assume the thickness, CTE, and Young's modulus of material in layer $i$ are $h_{i}, \alpha_{i}$, and $E_{i}$, respectively, and all layers have the same length $(L)$ and width $(b)$. Assuming the tip deflection at the free end of the structure $\delta$ is small compared to its length, it can be expressed as

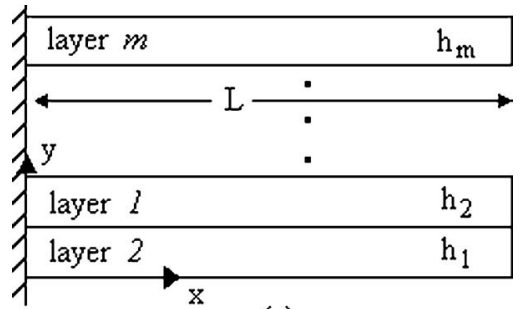

(a)

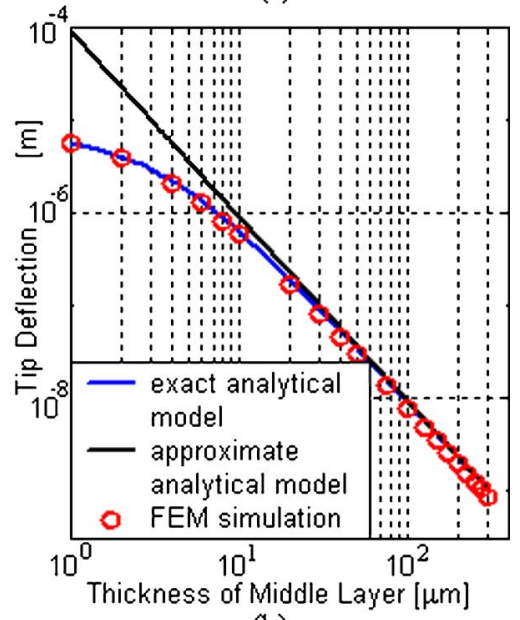

(b)

FIG. 1. (Color online) (a) One-end-fixed and one-end-free laminated layers have the same length $L$ along the $x$ axis and width $b$ along the $z$ axis. (b) Comparison of analytical models with the FEM simulation for deflection estimation for unity temperature difference induced on the cantilever. 


$$
\delta=\frac{1}{2 r} L^{2},
$$

where $r$ is the radius of curvature of the system. In the analysis, the only mechanism that drives the elastic deformation is assumed to be thermal strains developed in the layers of the structure as a result of temperature difference. The lengthy expression for $1 / r$ is given explicitly in Ref. 6 . For a threelayer system, the exact solution for $1 / r$ can be written as

$$
\frac{1}{r}=\frac{(A+B) \Delta T}{C+D+E+F},
$$

$A=6\left[E_{2} h_{2} h_{1}+E_{2} h_{2}^{2}+E_{3} h_{3}\left(h_{1}+2 h_{2}+h_{3}\right)\right] E_{1} h_{1}\left(\alpha_{2}-\alpha_{1}\right)$,

$B=6\left[E_{2} h_{2} h_{3}+E_{2} h_{2}^{2}+E_{1} h_{1}\left(h_{1}+2 h_{2}+h_{3}\right)\right] E_{3} h_{3}\left(\alpha_{3}-\alpha_{2}\right)$,

$C=E_{1} h_{1} E_{2} h_{2}\left(4 h_{1}^{2}+4 h_{2}^{2}+6 h_{1} h_{2}\right)$,

$D=E_{2} h_{2} E_{3} h_{3}\left(4 h_{1}^{2}+4 h_{3}^{2}+6 h_{2} h_{3}\right)$,

$E=E_{1} h_{1} E_{3} h_{3}\left[4 h_{1}^{2}+4 h_{3}^{2}+6 h_{1} h_{3}+12 h_{2}\left(h_{1}+h_{2}+h_{3}\right)\right]$,

$F=\sum_{1}^{3} E_{i}^{2} h_{i}^{4}$

The equation above can be used to analyze general three-layer structures including thin-film surfacemicromachined structures and bulk-micromachined structures encountered in MEMS. A case that is common in bulkmicromachined MEMS structures is that with a thick device layer, often silicon, and thin films on the top and bottom surfaces. For a three-layer structure with thick middle layer and thin films on the top and bottom surfaces [i.e., $h_{2}$ $\left.\gg\left(h_{1}, h_{3}\right)\right]$, we can find a first order approximate solution for $\delta$,

$$
\begin{aligned}
\delta= & {\left[3 E_{1} h_{1}\left(\alpha_{2}-\alpha_{1}\right)+3 E_{3} h_{3}\left(\alpha_{3}-\alpha_{2}\right)\right] \frac{L^{2} \Delta T}{E_{2} h_{2}^{2}} } \\
& \text { if } h_{2} \gg\left(h_{1}, h_{3}\right) .
\end{aligned}
$$

Setting $h_{1}$ or $h_{3}$ equal to zero in Eqs. (2) and (3) gives the solution for the bilayer case, which has been studied extensively in the literature. ${ }^{5,8,9}$ The above equation can be modified to obtain the solution for the case when the substrate layer is at the bottom and two film layers are on the top, which describes general optical microstructures, such as optical filters employing thin films on top of a thick substrate. If that is the case, the governing equation for thermal deflection is given as follows:

$$
\begin{aligned}
\delta= & {\left[3 E_{2} h_{2}\left(\alpha_{2}-\alpha_{1}\right)+3 E_{3} h_{3}\left(\alpha_{3}-\alpha_{1}\right)\right] \frac{L^{2} \Delta T}{E_{1} h_{1}^{2}} } \\
& \text { if } h_{1} \gg\left(h_{2}, h_{3}\right) .
\end{aligned}
$$

Figure 1(b) shows the agreement between the FEM and the exact and the approximate analytical solutions presented in Eqs. (2) and (3), assuming the thicknesses of $\mathrm{Au}$ and $\mathrm{Al}$ layers on opposite sides of a silicon cantilever, whose thickness varies in the range of $1-300 \mu \mathrm{m}$, are $2 \mu \mathrm{m}$ each. Note that there is very good agreement between the FEM and exact expression, and the error in the approximate solution is less than $20 \%$ when the thickness of the Si layer is more than ten times the film thicknesses, whereas the error drops to $2 \%$ when the Si layer is 100 times thicker than the metal films.

In the remainder of this paper, we focus on the first case where the thick substrate is the second layer. The second case can also be analyzed in a similar manner. For a parametric design study, we can define the dimensionless layer thickness ratio parameters $x=\left(h_{1} / h_{2}\right)$ and $y=\left(h_{3} / h_{2}\right)$ and rewrite Eq. (3),

$$
\delta=\left[3 E_{1} x\left(\alpha_{2}-\alpha_{1}\right)+3 E_{3} y\left(\alpha_{3}-\alpha_{2}\right)\right] \frac{L^{2} \Delta T}{E_{2} h_{2}} .
$$

The validation of the above approximation is presented in Fig. 1(b) for small $\delta$. Note that the deflection is proportional to the temperature difference and inversely proportional to the layer thicknesses if $x$ and $y$ are held constant. Setting Eq. (4) to zero gives the condition for an "athermal" three-layer design in which the surface flatness is insensitive to thermal changes.

$$
\frac{h_{3}}{h_{1}}=\frac{E_{1}\left(\alpha_{2}-\alpha_{1}\right)}{E_{3}\left(\alpha_{2}-\alpha_{3}\right)} \text {. }
$$

The above equation is simple yet powerful for the design of athermal structures in a compact form with defined materials. Equation (5) can be rewritten by using normalized parameters that also incorporate the material specific parameters $E$ and $\alpha$,

$$
\begin{aligned}
& w=\frac{E_{1}}{E_{2}} \frac{h_{1}}{h_{2}}\left(\alpha_{2}-\alpha_{1}\right), \quad z=\frac{E_{3}}{E_{2}} \frac{h_{3}}{h_{2}}\left(\alpha_{3}-\alpha_{2}\right), \\
& \delta=[w+z] \frac{3 L^{2} \Delta T}{h_{2}} .
\end{aligned}
$$

The unit of $w$ and $z$ is $\left(1 /{ }^{\circ} \mathrm{C}\right)$. If the materials are chosen such that $w=-z$, then the structure becomes athermalized.

\section{FEM AND EXPERIMENTAL RESULTS}

In this section, the analytical results given above for Fig. 1 are tested using a seemingly different three-layer MEMS scanner structure, illustrated in Fig. 2, which is a three-layer mirror plate connected to a rigid frame with two flexures. As discussed below, the analytical formulas derived for a fixedfree cantilever beam can be applied with some care to the geometry in Fig. 2.

The structure is bulk micromachined using single crystal silicon. The top surface is coated with an aluminum layer for high reflectivity and the bottom surface has an electroplated single layer gold coil with multiple turns with narrow gaps in between. The thin layer of Au from the center of the structure for the coil's electrical return path, the isolation oxide layer, and the metal plating base layers are all very thin compared to the coils; their effects on thermal deformation are neglected. The microscanner is actuated electromagnetically using an external magnetic field. When current flows in the coil, the magnetic flux density ( $B$ field) exerts a Lorentz 


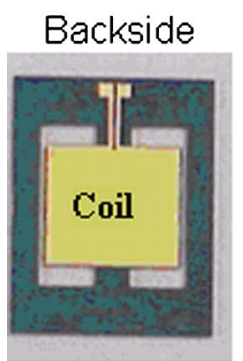

(a)

\section{Frontside}

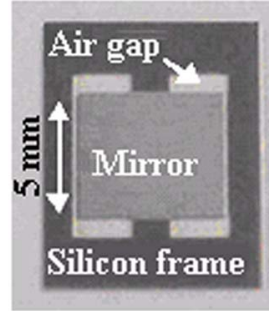

(b)

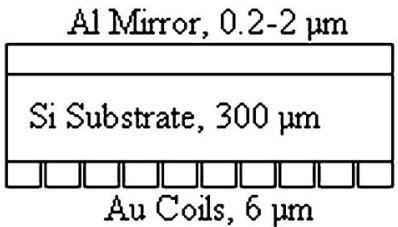

(c)
FIG. 2. (Color online) Torsional raster pinch scanner (a) back and (b) front sides, and (c) cross-sectional view illustrating three-layers and thicknesses.

force with opposite directions on either side of the coil, creating a net torque for torsional actuation of the microscanner. ${ }^{1}$ Thermal stresses and deformation in this structure can be significant as the structure heats up due to the resistive power dissipation on the coils. Since the aluminum side is used as a mirror, the flatness must be maintained to within one-tenth the wavelength of the light used $(\lambda / 10)$ under different operating temperatures and different coil current levels. ${ }^{10}$

Table I shows CTE and Young's modulus $(E)$ data for silicon, gold, and aluminum used in the experiments. The substrate layer is $300 \mu \mathrm{m}$ single crystal silicon (layer 2). Au (layer 1) and $\mathrm{Al}$ (layer 3) thicknesses can be adjusted for optimal actuator and thermal performance. Thermal sensitivity of the structure can be adjusted by adjusting layer thicknesses according to Eq. (3).

Mirror deformation was measured with an optical interferometer while applying direct current $(\mathrm{dc})$ to the coils without the external magnets. The scanner remains still but heats up due to resistive power dissipation and the temperature is measured using thermocouples. The zero-stress temperature for the structure is near the deposition temperature of the coils, which is around $35^{\circ} \mathrm{C}$. Figure 3 shows the mirror thermal deformation results for two different scanner dies: design 1 with $0.2 \mu \mathrm{m}$ and design 2 with $2 \mu \mathrm{m} \mathrm{Al}$ on the top surface. For design 1, when the power dissipated at the coils is $245 \mathrm{~mW}$, the coils heat up an additional $\Delta T=46.5^{\circ} \mathrm{C}$ from its zero-stress temperature and the deflected mirror shape is as shown in Fig. 4(a) with a maximum peak-tovalley (p-v) deflection of $0.51 \mu \mathrm{m}$ observed at the corners. The finite element method simulation result for the same

TABLE I. Material properties and thicknesses for three layer structure.

\begin{tabular}{cccc}
\hline \hline Material & $\begin{array}{c}\mathrm{CTE} \alpha \\
\left(10^{-6} \mathrm{~K}^{-1}\right)\end{array}$ & $\begin{array}{c}E \\
(\mathrm{GPa})\end{array}$ & $\begin{array}{c}h \\
(\mu \mathrm{m})\end{array}$ \\
\hline $\mathrm{Si}$ & 2.6 & 162 & 300 \\
$\mathrm{Al}$ & 25 & 69 & 0.2 and 2 \\
\hline \hline
\end{tabular}

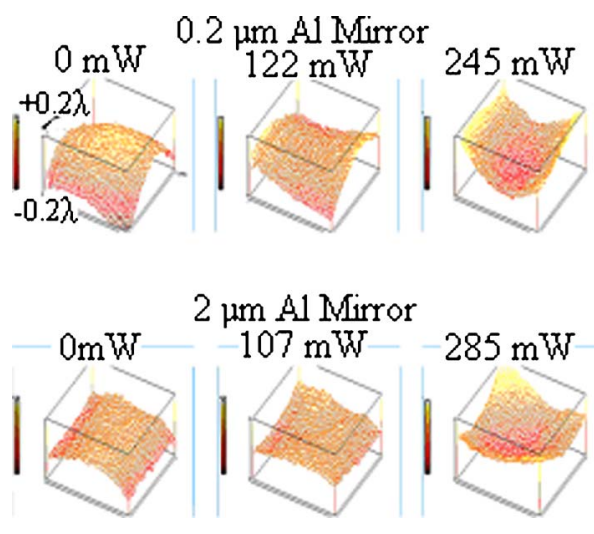

FIG. 3. (Color online) Interferometrically measured mirror flatness for two scanners with different mirror thicknesses. The top three figures are obtained for a $0.2 \mu \mathrm{m} \mathrm{Al} \mathrm{mirror} \mathrm{case;} \mathrm{the} \mathrm{bottom} \mathrm{three} \mathrm{figures} \mathrm{are} \mathrm{obtained} \mathrm{for} \mathrm{a}$ $2 \mu \mathrm{m} \mathrm{Al} \mathrm{mirror} \mathrm{case.} \mathrm{All} \mathrm{graphs} \mathrm{have} \mathrm{a} \mathrm{scale} \mathrm{of} \pm 0.2$ waves of optical path difference in the measurement setup.

case is given in Fig. 4(b) and shows a maximum p-v deflection of $0.54 \mu \mathrm{m}$ at the corners, which is in very good agreement with the experimental result.

Analytical results obtained in Sec. II can be compared with FEM and experimental results along the three crosssectional paths $\mathrm{a}, \mathrm{b}$, and $\mathrm{c}$ defined on (b). Note that the analytical formulas assume one-end-fixed and the other end-free beam boundary conditions. Furthermore, beam width effects and biaxial stress effects are not considered in the analysis. None of those assumptions seems valid for the structure analyzed; however, our numerical and experimental results show that the analytical results are still adequate. Using the biaxial modulus instead of Young's modulus seem more appropriate

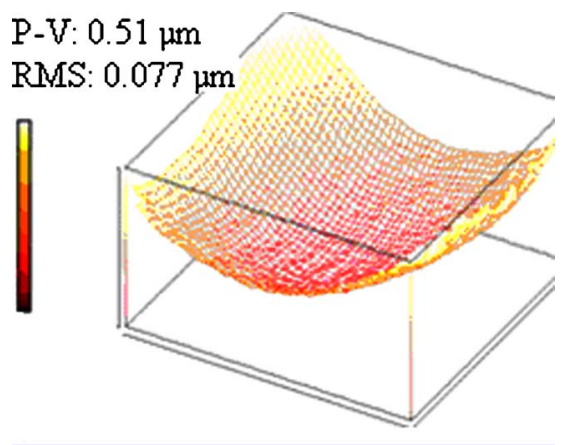

(a)

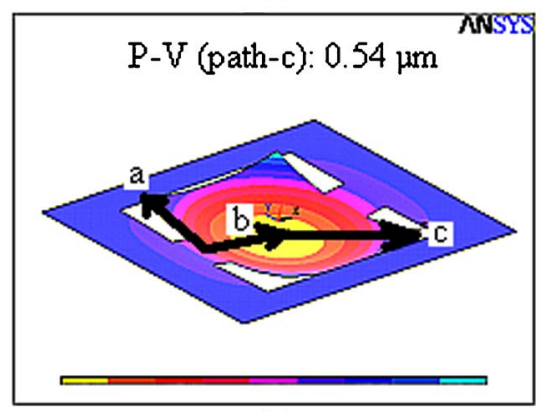

(b)

FIG. 4. (Color online) Thermal deformation for $\Delta T=46.5{ }^{\circ} \mathrm{C}$ temperature rise for structure in Fig. 2(a). (a) Interferometer measurement result, only the mirror surface is shown, and (b) FEM simulation result using ANSYSTM including the mirror and the frame. 


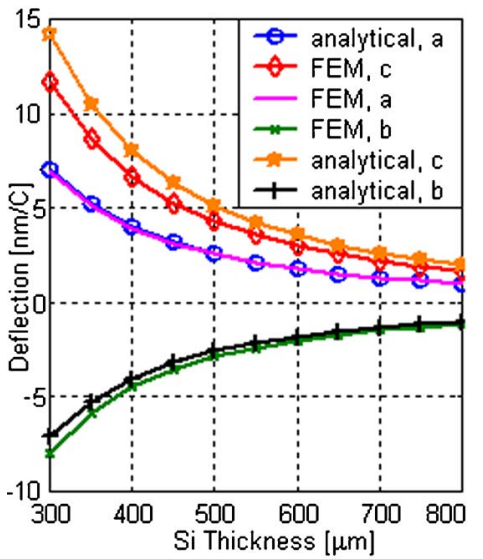

FIG. 5. (Color online) Comparison of the deflection amplitude between analytical solution and FEM a, b, and c. Note that, for $\Delta T>0, \delta_{\mathrm{a}}, \delta_{\mathrm{c}}>0$ and $\delta_{\mathrm{b}}<0$ for $0.2 \mu \mathrm{m} \mathrm{Al}$ case.

for wide beams; ${ }^{6}$ however, since the structure is anchored by narrow torsional flexures in this particular case, the use of $E$ in the analysis gives better results. For a rigorous analysis the deformation of the plate suspended by two flexures should be studied but a closed-form solution cannot be obtained. Experimental results for the effect of the plate area on the tip deflection are presented in Ref. 9.

Using the paths defined on the figure, the deflection of any point on the structure surface can be calculated by the weighted vector summation of the deflection along the paths. For example, deflection along path c can be visualized as the difference of deflections along path $b$ from path a. Figure 5 shows a comparison between the FEM and the approximate analytical solutions obtained for design 1 where Si thickness is a parameter. Deformation decreases with the square of the silicon thickness as predicted by Eq. (3). Analytical and FEM results agree very well along path a as the fixed-free beam assumption is valid, even though the beam width effects are ignored. We can assume that the center of the structure acts like a guided point due to the symmetry of the structure. Along path $\mathrm{b}$, the analytical prediction for the deflection amplitude is smaller by $13 \%$ (note the sign of deflection is negative in this case). This error is expected as the scanner cross section behaves as a fixed-guided beam from anchor to center of the mirror while our formulas predict the deformation for a fixed-free beam. For path $\mathrm{c}$, the analytical predictions are larger by $18 \%$. The error is again expected as the scanner cross section behaves as a guided-free beam from center to corner of the mirror while our formula predictions are for a fixed-free beam. For similar structures with different materials or dimensions, the calculated error factors can be added to the analytical predictions to improve the accuracy of the analytical predictions.

Figure 6 shows the $p-v$ surface deformation (i.e., deviation from flatness) as a function of temperature for both designs and demonstrates that FEM and experimental results agree well. The analytical predictions (calculated along path c) are larger than FEM predictions by $20 \%$ due to the approximations made by modeling the three-dimensional (3D) structure by a fixed-free simple beam. The device has some initial deformation due to stress and due to the deposition

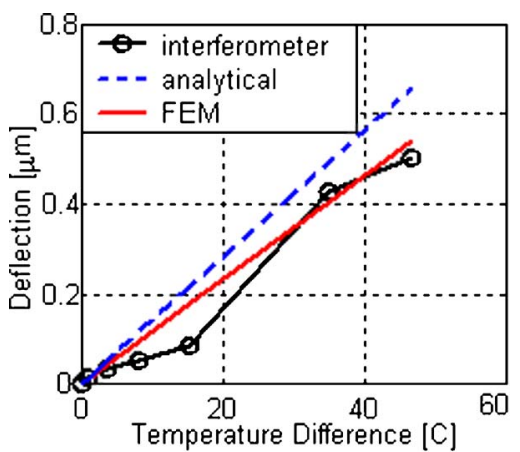

(a)

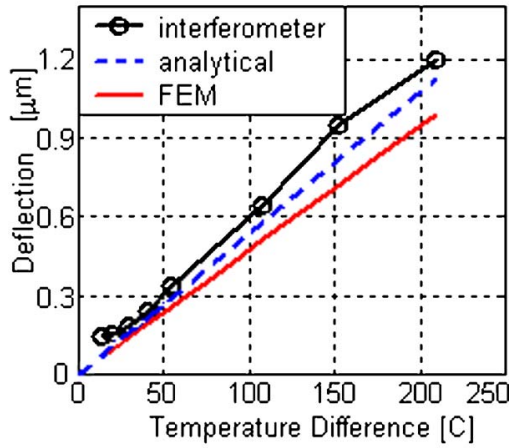

(b)

FIG. 6. (Color online) Comparison of thermal deflection as a function of temperature difference (measured with a thermocouple) for analytical solution along path c, FEM simulation, and interferometric measurement results for (a) $0.2 \mu \mathrm{m}$ and (b) $2 \mu \mathrm{m}$ case.

temperature, which is $15-20{ }^{\circ} \mathrm{C}$ above the room temperature. Therefore, as the structure is heated above the room temperature, the surface deformation attains a minimum near the film deposition temperatures and then the deformation changes sign and increases with temperature. Therefore, the agreement between FEM and the experimental results is better for high temperatures.

Figure 7(a) illustrates the analytical thermal deflection results using the closed-form exact analytical solutions for a large range of $\mathrm{Au}$ and $\mathrm{Al}$ thickness values assuming $\mathrm{Si}$ is $300 \mu \mathrm{m}$ and the scanner area is $5 \times 5 \mathrm{~mm}^{2}$ (i.e., $L=2.5 \mathrm{~mm}$ ). Film thickness larger than $10 \mu \mathrm{m}$ is hard to achieve with standard deposition methods. If all the thicknesses in the structure are multiplied by a scaling factor, the figure is still usable but the contour line values need to be divided by the same scaling factor. If the size of the scanner changes, then contour values should be scaled with $(L / 2.5)^{2}$, where $L$ $=2.5 \mathrm{~mm}$ is the assumed path length for the figure.

Figure 7(b) shows the exact solution given in Eq. (2) and approximate analytical solutions given in Eq. (3) for a thick $\mathrm{Si}$ structure sandwiched in between thin $\mathrm{Al}$ and $\mathrm{Au}$ films. Note that the approximate formula works well for thin films, particularly around the athermal line. The figure shows that the approximation error is small if the film to substrate thickness ratios are less than about $1 / 30$.

In our application, such a tradeoff helped improve the performance of the microscanner. Design 1 had $6 \mu \mathrm{m} \mathrm{Au}$ coils and $0.2 \mu \mathrm{m} \mathrm{Al}$, which is sufficient for good reflectivity. Later the Al thickness was increased to $2 \mu \mathrm{m}$ in design 2, which reduced the thermal deformation from +7.5 to about 


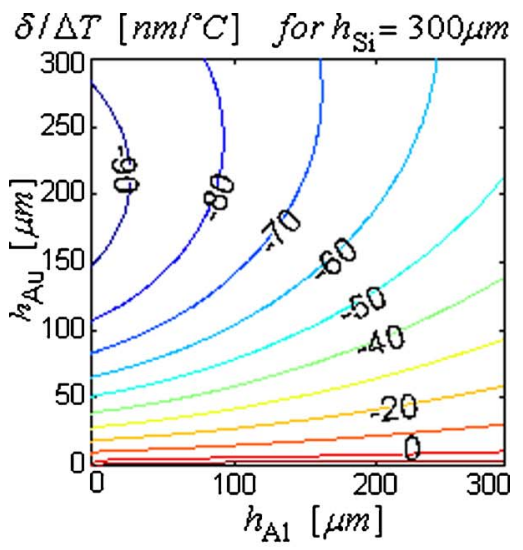

(a)

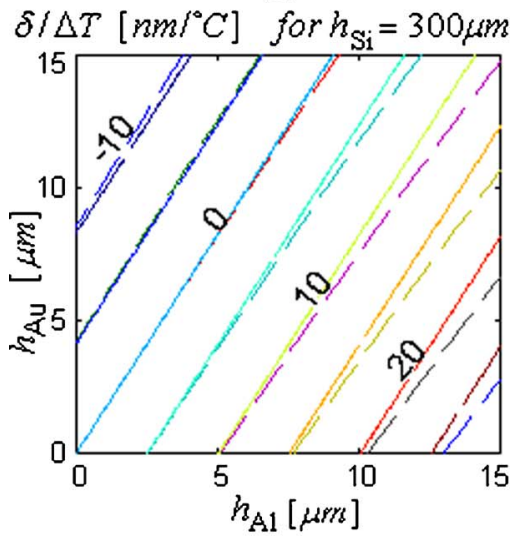

(b)

FIG. 7. (Color online) Thermal deformation of the scanner vs metal layer thicknesses (a) for thick films. (b) Comparison of exact and approximate expressions for thin films. The linear contour lines show the approximate solution and the nonlinear contour lines show the exact solution.

$1 \mathrm{~nm} /{ }^{\circ} \mathrm{C}$. If the $\mathrm{Al}$ thickness is increased to about $3.6 \mu \mathrm{m}$, the design falls on the athermal line in Fig. 7(b) and becomes completely athermal.

Figure 8(a) illustrates a dimensionless plot using the approximate deformation given in Eq. (5) using the thickness ratios $x$ and $y$. The contour labels show the deformation normalized with Si thickness, temperature, and scan mirror half diameter. The figure allows for easy performance trades for the three-layer structure used in our case study. For our design dimensions $h_{\mathrm{Si}}=300 \mu \mathrm{m}$ and $L=2.5 \mathrm{~mm}$, and assuming $\mathrm{Au}$ and $\mathrm{Al}$ film thicknesses are both chosen as $6 \mu \mathrm{m}$ (i.e., $x=y=0.02$ ), the thermal deformation can be calculated using the contour value of $2 \times 10^{-7}$,

$$
\delta / \Delta T=2 \times 10^{-7} \frac{\left(2.5 \times 10^{-3}\right)^{2}}{300 \times 10^{-6}}=4.2 \mathrm{~nm} /{ }^{\circ} \mathrm{C} .
$$

Figure 8(b) uses the coefficients $w$ and $z$ defined in Eq. (7) and shows the normalized thermal deformation $\left(\delta h_{2}\right) /\left(\Delta T L^{2}\right)$ using the approximate analytical solution. The figure is usable for an arbitrary selection of three-layer materials and thickness ratios assuming the middle substrate layer is thick compared to the film layers.

\section{CONCLUSIONS}

We presented exact and approximate analytical solutions to calculate the thermally induced deformation for three-

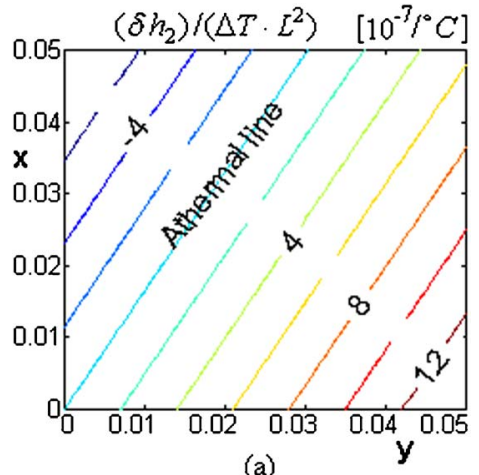

(a)

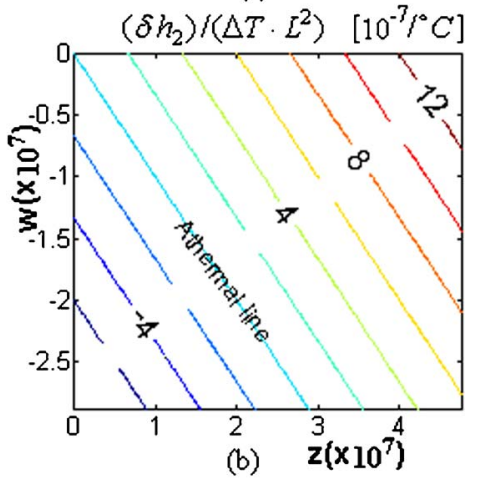

FIG. 8. (Color online) Normalized thermal deformation contour plots for three-layers using approximate analytical formulas. (a) Using film to substrate thickness ratios $x$ and $y$ as parameters for $\mathrm{Al}, \mathrm{Si}$, and $\mathrm{Au}$ based structures. (b) Using the normalized coefficients $w$ and $z$ as parameters. The figure is usable for any material combination.

layer structures. Analytical results are applied to estimate the deformation for a square-shaped three-layer MEMS scanner structure suspended by two torsional flexures. Even though the formulas are derived for cantilever beams, the error for the maximum thermal deformation in the MEMS scanner is only about $18 \%$ compared to FEM results. The maximum discrepancy between analytical, FEM, and experimental results is observed to be less than $30 \%$. The approximate analytical formulas are found to be valid if the film thickness is less than 1/30 of the substrate thickness. In our design study, the analytical results are employed to make the scanner structure nearly athermal by making the Al layer thicker. A useful set of figures is laid out, which can be modified for different material combinations to do different performance trades in order to make the structure less or more deflecting in response to thermal changes. Making the structure athermal is possible with the right combination of materials and thicknesses.

\section{ACKNOWLEDGMENT}

One of the authors (H.T.) is now a Ph.D. student of Georgia Institute of Technology.

${ }^{1}$ A. D. Yalcinkaya, H. Urey, D. Brown, T. Montague, and R. Sprague, IEEE/ASME J. Microelectromech. Syst. 15(4) (2006).

${ }^{2}$ L. Wook, N. A. Hall, Z. Zhiping, and F. L. Degertekin, IEEE J. Quantum Electron. 10, 643 (2004).

${ }^{3}$ P. G. Datskos, N. V. Lavrik, and S. Rajic, Rev. Sci. Instrum. 75, 1134 (2004). 
${ }^{4}$ H. Torun and H. Urey, Opto-Electron. Rev. 14, 55 (2006).

${ }^{5}$ S. Timoshenko, J. Opt. Soc. Am. 11, 233 (1925).

${ }^{6}$ C. H. Hsueh, J. Appl. Phys. 91, 9652 (2002).

${ }^{7}$ G. P. Nikishkov, J. Appl. Phys. 94, 5333 (2003).
${ }^{8}$ C. Hsueh, J. Appl. Phys. 91, 9652 (2002).

${ }^{9}$ Y. Y. Hu and W. M. Huang, J. Appl. Phys. 96, 4154 (2004).

${ }^{10} \mathrm{H}$. Urey, in Encyclopedia of Optical Engineering, edited by R. Driggers (Dekker, New York, 2003), Vol. 3, pp. 2445-2457. 